\title{
Magnetic resonance imaging of the left wrist: assessment of the bone age in a sample of healthy Iraqi adolescent males
}

\author{
Najah R. Rashid* \\ Mohammed Al-Hilli* \\ Abdullateef Aliasghar** \\ Qasim M. Shaker***
}

\author{
MBChB, CABMS (RAD) \\ MBChB, DMRD, FICMS (RAD) \\ MBChB, CABMS (RAD) \\ MBChB, FICMS (ORTHO)
}

\begin{abstract}
:
Background: Use of magnetic resonance imaging (MRI) to calculate skeletal age is a novel idea. MRI provides excellent soft-tissue contrast and multiplanar cross-sectional imaging capability. It could be used as an alternative method of skeletal age determination.

Objectives: To study the value of MRI in estimating the age of healthy Iraqi adolescent males and to compare the obtained results with other countries records.

Fac Med Baghdad 2015; Vol.57, No.1 Received: July, 2014 Accepted: Jan., 2015

Population and methods: This cross sectional study was applied on 179 healthy adolescent males between the ages of 13 to 18 years in MRI unit at radiology institute in medical city, Baghdad - Iraq. This study was carried out from November 2011 to December 2012. Magnetic resonance imaging of the left wrist was performed by using a 1.5 Tesla machine with surface coil. The sequence used was coronal T1weighted images (WI). The degree of fusion of the left distal radial physis was determined by a newly developed grading system.

Results: There is high correlation between chronological age and degree of fusion of distal radius within the participant population. Most adolescent boys in the age group between 13 and 14 years presented as grade I and II, while the complete fusion was found at the age of 17 and 18 years, the mean age of participants was 17.5 years. Degree of fusion of the distal radius in the sample of the study was almost approaching the obtained values in the Algeria and Malaysia as comparative countries.

Conclusion: MRI offers an alternative; non-invasive method of examination of the epiphysial fusion, which eliminates any risk associated with standard radiographic rating. The grading system can accurately identify the variable degrees of epiphysial fusion in an objective teachable manner.

Key words: bone age, magnetic resonance imaging, left wrist.
\end{abstract}

\section{Introduction:}

Skeletal age is defined as the age at which normal children of same sex generally achieve the skeletal maturation stage for determined chronological age. Thus, skeletal age should correspond to the chronological age in healthy children of same sex in reaching a similar degree of skeletal development.1 Defining an individual's correct age is important in medicolegal practice 2 and sport medicine.3 Standard radiography analyses of the left wrist have been used for decades to estimate the age and potential to grow following the published standards established by Greulich and Pyle, Tanner and Fels.4,5,6 The appearance of different degrees of fusion of the distal radius epiphysial growth plate using magnetic resonance imaging, a radiation-free imaging modality that can be used in healthy people such as athletes and football players.7

Bone age assessment is a procedure frequently performed in

*Radiologist - Medical city complex - Baghdad.

**Corresponding Auther: Abdullateef Aliasghar National Center for

Cancer Research - University of Baghdad.

E-mail: latifashoor@yahoo.com

***Orthopedic Surgeon-Medical city complex - Baghdad. pediatric radiology. Based on a radiological examination of skeletal development of the left-hand wrist, bone age is assessed and then compared with the chronological age. A discrepancy between these two values indicates abnormalities in skeletal development. The procedure is often used in the management and diagnosis of endocrine disorders and it can also serve as an indication of the therapeutic effect of treatment. Generally, it can indicate whether the growth of a patient is accelerating or decreasing. 8

MRI is radiation-free with superior imaging ability, which can be used to image the epiphyses of the ulna and radius. Using MRI, additional information regarding the ossification centers of the epiphyses may be obtained. A clear difference between the ossification centers according to age was observed.9 A new MRI grading classification of growth plate fusion of distal radius has been established.10

The main objectives of this study are to determine skeletal bone age in the age group 13-18 years and to assess a grading system of magnetic resonance imaging (MRI) for epiphysial fusion of the distal radius in a sample of healthy Iraqi adolescent male 
and compare our results with outcomes obtained in different countries.

\section{Population and methods}

This cross sectional study was done during the period from November 2011 to December 2012 and applied on 179 healthy adolescent males between the ages of 13 and18 years at MRI unit, radiology institute, medical city, Baghdad-Iraq. MR imaging study of the left wrist performed by using a $1.5 \mathrm{~T} \mathrm{MR}$ Machine (Siemens healthcare, Germany) with surface coil. The sequence used was coronal T1WI FSE. The degree of fusion of the left distal radial physis determined by a newly developed grading system. Inclusion criteria includes healthy adolescent Iraqi male participant his age ranges from 13-18 years; while Exclusion criteria includes participants unable to produce the original or verified copy of their birth certificate or identity card, participants who are claustrophobic and have a history of ferro-magnetic implants and or indwelling metallic intraocular foreign bodies, participants known to have history of genetic, developmental, metabolic, nutritional or endocrinal diseases, which may affect bone growth, participants with known history of trauma to the wrist which can cause growth arrest or premature total fusion of the epiphysis, participants who are or were on medication or hormonal supplements which may affect skeletal growth and female participants. Oral consent was obtained from each of the participants before have their clinical data was recorded and MRI examination performed and all participants were provided verbal explanation of the purpose of the study.

Population: Young healthy adolescent Iraqi male from Baghdad aged between 13 and 18 years were selected by regional football clubs and schools. The sample of study grouped according to their birth certificates into: $13 \mathrm{Y}, 14 \mathrm{Y}$, $15 \mathrm{Y}, 16 \mathrm{Y}, 17 \mathrm{Y}, \& 18 \mathrm{Y}$. Then the MRI protocol applied and classified the study population into 6 grades according to their ossification and fusion of left distal radius on MR images as follow: Grade I: Completely unfused. Grade II: Early fusion: minimal hyperintensity within the physis. Grade III: Trabecular fusion of $<50 \%$ of the radial crosssectional area. Grade IV: Trabecular fusion of $>50 \%$ of the radial crosssectional area. Grade V: Residual physis, $<5 \mathrm{~mm}$ on any one section.

Grade VI: Completely fused. All these MRI findings are evaluated by two board-certified radiologists.

MRI protocol: The examination protocol was applied using MRI equipment; the wrist was positioned above the head or at the side of the body. The third metacarpal was placed as close as possible to the same axis as the radius. Coronal sequences were planned parallel to the distal volar radial surface. The following parameters were applied: T1-weighted spin echo, TR 350-500 ms, TE 12-20 ms, flip angle 90o, slice thickness $3 \mathrm{~mm}$, interslice gap $0.3 \mathrm{~mm}$ (1.1 distance factor), matrix (256 x 256), FOV (100), pixel size (0.5 mm), 2-4 acquisitions and 9 images (to cover the entire distal radius from anterior to posterior). Nine images were included per film, and then printed on CDs with the wrist enlarged so as to include the distal $3 \mathrm{~cm}$ of the radius, and the entire corpus was included. TA (5 min, $48 \mathrm{sec})$.

Statistical analysis: All the findings studied were tabulated using Microsoft Excel hence statistical analysis was done by using Statistical packages SPSS18 (Statistical Package for Social Sciences version 18). Continuous variables presented and discrete variables presented as numbers and percentages.
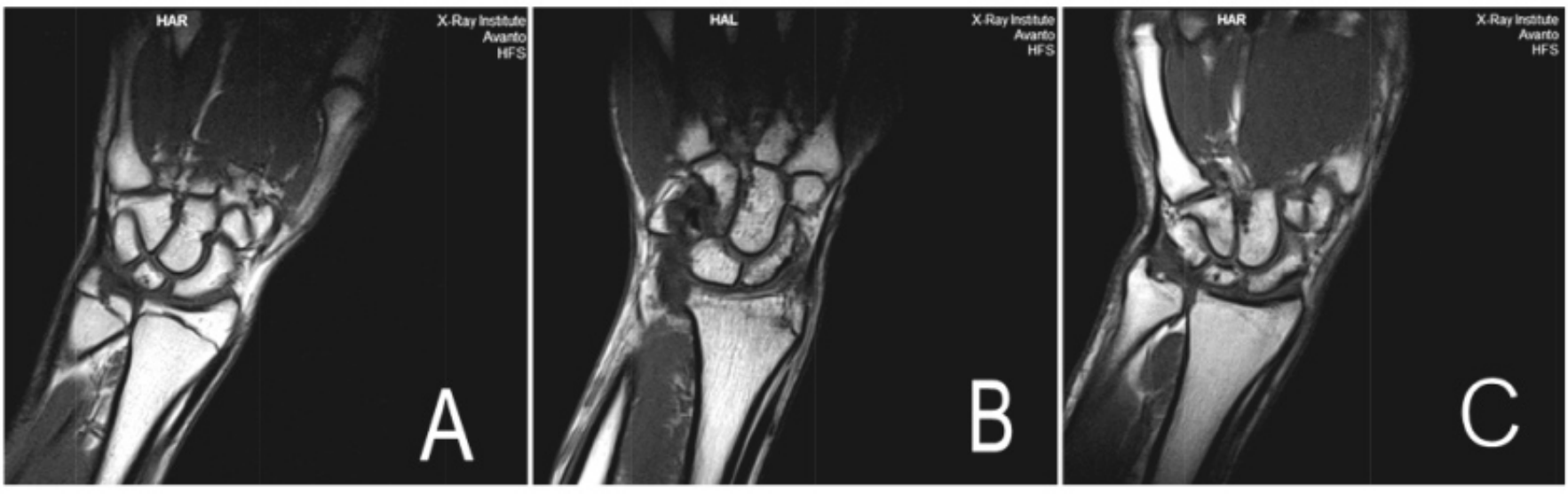

Figure (1) A. Grade- III: T1-weighted spin echo image of distal left radius showing trabecular fusion of less than $50 \%$ of the radial cross-sectional area. B. Grade- IV: T1-weighted spin echo image of distal left radius showing trabecular fusion of more than $50 \%$ of the radial cross-sectional area. C. Grade- VI: T1-weighted spin echo image of complete fusion of distal left radius. 


\section{Results}

Table (1) Age distribution of sample of study according to birth certificates issued by governmental institutions.

\begin{tabular}{ccc}
\hline Age group (year) & Number & Percentage \\
\hline 13 & 29 & $16.2 \%$ \\
\hline 14 & 27 & $15 \%$ \\
\hline 15 & 33 & $18.4 \%$ \\
\hline 16 & 29 & $16.2 \%$ \\
\hline 18 & 32 & $18 \%$ \\
\hline Total & 29 & $16.2 \%$ \\
\hline
\end{tabular}

A total of 179 participants with self-reported ages of 13- 18 years presented for the MRI examination of the left wrist. The mean age of the participants was 16.6 years. In total, 179 participants were examined during the study; 29 (16.2\%) were 18 years old and $32(18 \%)$ were 17 years old and $118(65.8 \%)$ were 16 years old or below as shown in table (1).

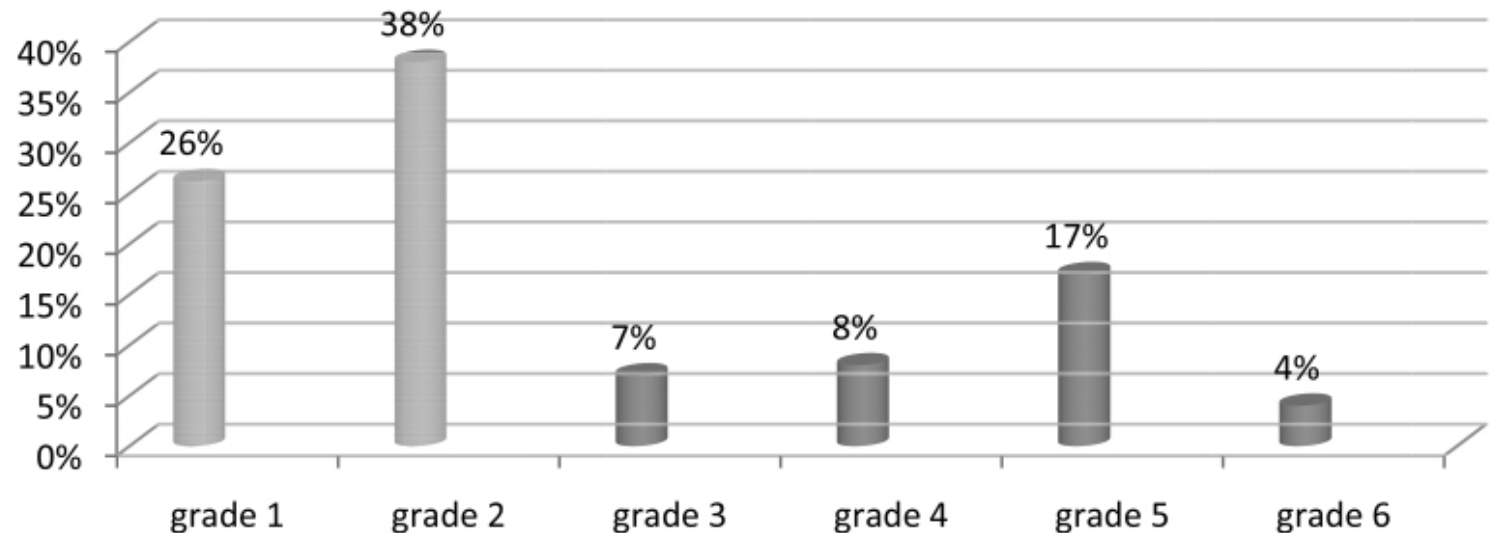

Figure (2) MRI- grade distribution of sample of study

Figure (2) shows the distribution of grades of fusion of the distal radius in different age groups of participants. Seven (4\%) participants had complete fusion (grade-6) and 30 (17\%) of participants was graded as 5 .

Table (2) Number of participants in relation to grading of fusion in the different age groups

\begin{tabular}{cccccccc}
\hline Grades & 13 years & 14 years & 15 years & 16 years & 17 years & 18 years & Total \\
\hline Grade 1 & $18(61 \%)$ & $14(53 \%)$ & $11(32 \%)$ & $3(11 \%)$ & $1(4 \%)$ & 0 & 47 \\
\hline Grade 2 & $11(39 \%)$ & $13(47 \%)$ & $16(49 \%)$ & $16(53 \%)$ & $10(32 \%)$ & $2(6 \%)$ & 68 \\
\hline Grade 3 & 0 & 0 & $3(10 \%)$ & $3(11 \%)$ & $3(9 \%)$ & $3(9 \%)$ & 12 \\
\hline Grade 4 & 0 & 0 & $2(6 \%)$ & $3(11 \%)$ & $6(18 \%)$ & $4(15 \%)$ & 15 \\
\hline Grade 5 & 0 & 0 & $1(3 \%)$ & $4(13 \%)$ & $9(27 \%)$ & $16(58 \%)$ & 30 \\
\hline Grade 6 & 0 & 0 & 0 & 0 & $3(10 \%)$ & $4(12 \%)$ & 7 \\
\hline Total & 29 & 27 & 33 & 29 & 32 & 179 \\
\hline
\end{tabular}


Table (2) shows the cross-tabulation of self-reported chronological age and degree of fusion. Seven participants presented as grade- 6,4 of them were belong in 18 years and 3 of them in the 17 years; no complete fusion of distal radius epiphysis reported in the 16 years or below.

Table (3) Number of participants and mean age in different categories of fusion grading

\begin{tabular}{ccc}
\hline Grade & Number of participant & mean age \\
\hline 1 & 47 & 15 \\
\hline 2 & 68 & 15.5 \\
\hline 3 & 12 & 16.5 \\
\hline 4 & 15 & 16.5 \\
\hline 5 & 30 & 16.5 \\
\hline 6 & 7 & 17.5 \\
\hline
\end{tabular}

Table (3) reveals the grading of fusion in relation to mean age. The average age increased with a higher grading of fusion.

\section{Discussion:}

MRI of distal radius fusion is currently being used in the world cup to exclude overaged players.1 Dovark et al suggested age determination with MRI offers an alternative as a noninvasive method of examination of epiphyseal fusion. MRI grading system presented clearly identifies different degrees of epiphysial fusion of the distal radius.11 The need for an alternative method of determining age and maturity has been raised by the International Atomic Energy Agency regulatory body, which does not allow x-ray examination except when clinically justified for the individual, which is not the case for age determination in sports or even in medico-legal situations except when a court order exists, based on criminal charges. 10 The determination of skeletal maturity is crucial in the practice of pediatrics, especially in relation to endocrinological problems and growth disorders. Age is also decisive for the punishment of delinquents in a court of law and competitive sport. Standard radiograph analyses of the left wrist have been used for decades to estimate the age and potential to grow following the published standards established by Greulich and Pyle4, Tanner and Fels.5,12, 13 There is high correlation between age and fusion within general population. Most adolescent boys in the age group between 13 and 14 years presented as grade I and II. Complete fusion occurs at the age of 17-18 years, which is in agreement with Dvorak et al.10.

Table (4) Mean age-dependent grading of fusion in different countries

\begin{tabular}{ccccccccccccc}
\hline Country & Grade 1 & \multicolumn{2}{c}{ Grade 2 } & \multicolumn{2}{c}{ Grade 3 } & \multicolumn{2}{c}{ Grade 4 } & \multicolumn{2}{c}{ Grade 5 } & Grade 6 \\
\hline Iraq & 15 & $\mathrm{~N}=47$ & 15.5 & $\mathrm{~N}=68$ & 16.5 & $\mathrm{~N}=12$ & 16.5 & $\mathrm{~N}=15$ & 16.5 & $\mathrm{~N}=30$ & 17.5 & $\mathrm{~N}=7$ \\
\hline Algeria & 15.7 & $\mathrm{~N}=29$ & 16.7 & $\mathrm{~N}=52$ & 16.7 & $\mathrm{~N}=11$ & 17.2 & $\mathrm{~N}=7$ & 18.1 & $\mathrm{~N}=18$ & 18.9 & $\mathrm{~N}=3$ \\
\hline Malaysia & 15.9 & $\mathrm{~N}=7$ & 16.1 & $\mathrm{~N}=41$ & 16 & $\mathrm{~N}=10$ & 17.4 & $\mathrm{~N}=18$ & 18.4 & $\mathrm{~N}=36$ & 18.1 & $\mathrm{~N}=15$ \\
\hline
\end{tabular}

In our study, the mean age of participants with complete fusion of the radius was 17.5 years indicating that complete fusion is very unlikely to occur at 16 years of age, which is disagreement with Dvorak et al, where, the mean age was 18.3 years; in Algeria, the mean age was 18.9 years and 18.1 years in Malaysia. 10 as shown in table (4). The possibility of finding a grade 6 (complete fusion of growth plate) between 16 and 17 years of age is less than 1\% (99\% show non-fusion, i.e. grade 5 and below) as obtained in Dvorak et al 10, where as in our study there is no body have complete fusion in this age group. There are several limitations encountered in this study. First, the study population did not include female participants. Second, all participants are of Asian ethnic group. Third, the epiphyseal fusion grading evaluated only by single MRI pulse sequence (T1-weighted images); therefore, the possibility of another sequence in addition to T1- weighted images to determine the process of bone fusion of the distal radius epiphysis may be explore new findings.

\section{Conclusions:}

There is a considerable correlation between age and MRI grade of fusion of the distal radial growth plate. MRI examination of distal radius is useful tool for determining epiphysial fusion and degree of maturity. which eliminates any risk associated with standard radiographic rating. Degree of fusion of the distal radius in the sample of the study was almost approaching the obtained values in the Algeria and Malaysia as comparative countries. As the presented study did not register weight and height, we recommend including anthropometrical data including body mass index to analyse the possible influence on the speed of maturation.

\section{Author contributions:}

Najah Rahem Rashid, Abdullateef Aliasghar: Authors make substantial contributions to conception and design, and/or acquisition of data, and/or analysis and interpretation of data. Mohammed Al-Hilli, Qasim Mohammed Shaker: Authors participate in drafting the article or revising it critically for important intellectual content; authors give final approval of the version to be submitted.

\section{References:}

1. John $G$, Jayyaselvi $N$, Khairul A. comparison study of growth plate fusion MRI versus plain radiographs as used in age determination for exclusion of overaged football players. Br J Sports Med. 2012; 46(4):273-278. [IVSL] 
2. Schmitt A. Forensic Anthropology and Medicine: Complementary Sciences from Recovery to Cause of Death. New Jersey; 2006: 212-219.

3. Helsen WF, Van Winckle J, Williams AM. The relative age effect in youth soccer across Europe. J Sports Sci, 2005; 23:629-36.

4. Greulich WW, Pyle SI: Radiographic Atlas of Skeletal Development of the Hand and Wrist. Second ed. Stanford: Stanford Univ. Press; 1959.

5. Tanner JM: Growth at Adolescence. Second ed. Oxford: Blackwell; 1962.

6. Roche AF, Chumlea WC, Thissen D. Assessment of skeletal maturity of the hand- wrist: Fels method. Springfield, 1988; 78:39.

7. Yoshikazu S, Daisuke S, Jiro K, et al. comparison of $M R$ images for age determination; T1 weighted images (T1WI) versus $T 2 *$ weighted images $(T 2 * W I)$. Asian journal of sport medicine, 2012; 3(1):47-52. [IVSL]

8. Pietka, E, Gertych, A, Pospiech, S, et al. Computer-assisted bone age assessment: Image preprocessing and epiphyseal/ metaphyseal ROI extraction. IEEE Transaction on Medical Imaging, 2001; 20(8):715-729. [IVSL]

9. Kamilla S, Liat T. A comparison of two modalities for bone age assessment: Ultrasound and MRI. 2005; 54:11-16.

10. Dovark J, John G, Astrid J, et al. Age determination by magnetic resonance imaging of the wrist in adolescent male football players. Br J Sports Med 2007; 41:45-52. [IVSL]

11. Dovark J, John G, Astrid J, et a

12.l. Application of MRI of the wrist for age determination in international U-17 soccer competitions. Br J Sports Med 2007; 41:497-500. [IVSL]

13. Mora S, Boechat MI, Pietka E, et al. skeletal age determinations in children of European and African descent: applicability of Greulich and Pyle standards. Pediatric Res 2001; 50(5):624-628.

14. Serinelli $S$. Accuracy of three age determination X-ray methods on the left hand-wrist: a systematic review and metaanalysis. Leg Med (Tokyo) 2011;13(3):120-33. 